\title{
Genome analysis of Bacteroides sp. CACC 737 isolated from feline for its potential application
}

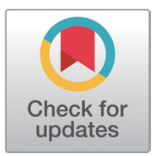

Received: Aug 4, 2020

Revised: Sept 21, 2020

Accepted: Oct 15, 2020

${ }^{*}$ Corresponding author

Yangseon Kim

Department of Research

and Development, Center for

Industrialization of Agricultural and

Livestock Microorganisms,

Jeongeup 56212, Korea.

Tel: +82-63-536-6712

E-mail: yangseon@cialm.or.kr

Copyright (C) 2020 Korean Society of

Animal Sciences and Technology.

This is an Open Access article

distributed under the terms of the

Creative Commons Attribution

Non-Commercial License (http://

creativecommons.org/licenses/by-

$\mathrm{nc} / 4.0 /$ ) which permits unrestricted

non-commercial use, distribution, and

reproduction in any medium, provided

the original work is properly cited.

ORCID

Jung-Ae Kim

http://orcid.org/0000-0002-0694-477X

Min Young Jung

http://orcid.org/0000-0001-9599-2345

Dae-Hyuk Kim

http://orcid.org/0000-0002-9948-5313

Yangseon Kim

http://orcid.org/0000-0002-8285-3407

\section{Competing interests}

No potential conflict of interest relevant

to this article was reported.

Funding sources

This research was supported the Strategic Initiative for Microbiomes in Agriculture and Food grant No.

918002-4, Ministry of Agriculture, Food and Rural Affairs, Korea and the Next-

\author{
Jung-Ae Kim ${ }^{1,2}$, Min Young Jung ${ }^{1}$, Dae-Hyuk Kim ${ }^{1,3}$ and Yangseon Kim ${ }^{1 *}$
}

${ }^{1}$ Department of Research and Development, Center for Industrialization of Agricultural and Livestock Microorganisms, Jeongeup 56212, Korea

${ }^{2}$ Department of Bioactive Material Sciences, Jeonbuk National University, Jeonju 54896, Korea

${ }^{3}$ Department of Molecular Biology, Jeonbuk National University, Jeonju 54896, Korea

\begin{abstract}
Bacteroides sp. CACC 737 was isolated from a feline, and its potential probiotic properties were characterized using functional genome analysis. Whole-genome sequencing was performed using the PacBio RSII and Illumina HiSeq platforms. The complete genome of strain CACC 737 contained $4.6 \mathrm{Mb}$, with a guanine $(\mathrm{G})+$ cytosine $(C)$ content of $45.8 \%$, six cryptic plasmids, and extracellular polysaccharide gene as unique features. The strain was beneficial to animal health when consumed as feed, for example, for ameliorating immunological dysfunctions and metabolic disorders. The genome information adds to the comprehensive understanding of Bacteroides sp. and suggests potential animal-related industrial applications for this strain.
\end{abstract}

Keywords: Bacteroides sp., Feline, Whole genome sequencing

\section{ANNOUNCEMENT}

Bacteroides species are gram-negative, anaerobic, non-spore-forming, bile-resistant bacteria that reside in the gut. They constitute approximately $25 \%$ to $30 \%$ of the intestinal gut microbiota of humans and other animals [1]. These bacteria have been proposed as next-generation probiotics by virtue of the action on the intestinal immune system [2]. In companion animals, Bacteroides associated with immune proteins, such as Tumor necrosis factor (TNF)- $\alpha$ and decreased the relative abundance with chronic enteropathy $[3,4]$.

We isolated Bacteroides sp. CACC 737 (KACC 22065) from the feces of a male 9-year-old Persian chinchilla in Korea. The sample was incubated in anaerobic atmosphere (5\% carbon dioxide, $5 \%$ hydrogen, and $90 \%$ nitrogen) at $37^{\circ} \mathrm{C}$ for $48 \mathrm{~h}$ on De, Rogosa and Sharpe (MRS) media. The isolate was considered to be a novel species of Bacteroides based on its $16 \mathrm{~S}$ rRNA sequence that displayed the highest similarity to the type strain B. uniformis ATCC8492T (97.5\%), which was below the suggested novel species recognition threshold of 98.6\% [5]. Genomic DNA was extracted from CACC 737 cell pellets using a DNeasy UltraClean microbial kit (QIAGEN, Hilden, Germany), consistent with the manufacturer's instructions. The isolated DNA was sequenced using single molecular real-time Portal (v2.3) with the PacBio RS II system (Pacific Biosciences, Menlo Park, CA, USA; Macrogen, Seoul, Korea). 
Generation BioGreen 21 Program (Project No. PJ01322304), Rural Development Administration, Korea.

Acknowledgements Not applicable.

Availability of data and material Upon reasonable request, the datasets of this study can be available from the corresponding author.

Authors' contributions

Conceptualization: Kim Y

Data curation: Kim JA.

Formal analysis: Jung MY.

Methodology: Kim JA.

Software: Jung MY.

Validation: Kim JA, Kim DH.

Investigation: Kim Y.

Writing - original draft: Kim JA, Kim Y.

Writing - review \& editing: Kim Y.

Ethics approval and consent to participate This article does not require IRB/IACUC approval because there are no human and animal participants.
The annotation of the genome sequences was carried out using the combined results of the automatic National Center for Biotechnology Information Prokaryotic Genomes Annotation Pipeline and the Rapid Annotations Subsystems Technology prokaryotic genome annotation server (http:// rast.nmpdr.org) [6]. The clustered regularly interspaced short palindromic repeats (CRISPR) were assessed using CRISPR web server (http://crispr.i2bc.paris-saclay.fr) [7,8].

Bacteroides species harbor cryptic plasmids at a high frequency (50\%) [9]. The complete genome of Bacteroides sp. CACC 737 genome revealed six cryptic plasmids ranging from 20 to $40 \mathrm{~kb}$ with an average GC content of $40.9 \%$ as well as a single circular chromosome of 4,470,359 bp with a GC content of $46.0 \%$ (Table 1 and Fig. 1A). The genome also contained 13 rRNAs and 69 transfer RNAs. A total of 3,938 protein-coding sequences (CDSs) were identified. Plasmids include hypothetical proteins and include genes involved in carbohydrate metabolism. Furthermore, 3,938 CDSs were specifically to clusters of 20 Clusters of Orthologous Groups of proteins (COGs)-based functional categories (Fig. 1B). Many genes were classified into functional categories for carbohydrate transport and metabolism $(n=270)$, cell wall/membrane/envelope biogenesis $(n=263)$, recombination and repair $(\mathrm{n}=231)$, inorganic ion transport and metabolism $(\mathrm{n}=227)$, amino acid transport and metabolism $(n=176)$, translation, ribosomal structure, and biogenesis $(n=151)$.

Two confirmed CRISPR regions (1 and 2) and one questionable CRISPR 9 region were detected. The pattern was identified as the CRISPR-CAS II type. The characterization of type II elements may reveal molecular genome editing tools for the development of next-generation probiotics [10]. The complete genome sequence of Bacteroides sp. CACC 737 will provide fundamental knowledge of the probiotic effects in host healthcare.

The complete genome of strain CACC 737 has been deposited to the National Center for Biotechnology Information GenBank database under accession numbers CP059408 (chromosome) and CP059406, CP059407, CP059409 - CP059412 (plasmids).

\section{REFERENCES}

1. Wexler HM. Bacteroides: the good, the bad, and the nitty-gritty. Clin Microbiol Rev. 2007;20:593-621. http://doi.org/10.1128/CMR.00008-07

2. Dahiya DK, Renuka, Dangi AK, Shandilya UK, Puniya AK, Shukla P. New-generation probiotics: perspectives and applications. In: Faintuch J, Fainguch S, editors. Microbiome and metabolome in diagnosis, therapy, and other strategic applications. Cambridge, MA: Academic Press; 2019. p. 417-24.

3. Xu H, Huang W, Hou Q, Kwok LY, Laga W, Wang Y, et al. Oral administration of compound probiotics improved canine feed intake, weight gain, immunity and intestinal microbiota. Front

Table 1. Genome overview of Bacteroides sp. CACC 737

\begin{tabular}{lccccccc}
\hline \multirow{2}{*}{ Attribute } & Chromosome & \multicolumn{7}{c}{ Plasmids } \\
\cline { 3 - 7 } & & $\mathbf{1}$ & $\mathbf{2}$ & $\mathbf{3}$ & $\mathbf{4}$ & $\mathbf{5}$ \\
\hline Size $(\mathrm{kb})$ & 4,470 & 29 & 22 & 40 & 23 & 29 & $\mathbf{6}$ \\
GC\% & 45.96 & 40.69 & 41.13 & 44.75 & 39.87 & 40.88 & 38.36 \\
Protein & 3761 & 31 & 25 & 39 & 35 & 31 & - \\
rRNA & 13 & - & - & - & - & - \\
tRNA & 65 & 1 & - & 3 & - & - \\
Acession No. & CP 059408 & CP 059406 & CP 059407 & CP 059409 & CP 059410 & CP 059411 \\
\hline
\end{tabular}

GC, guanine-cytosine; rRNA, ribosomal RNA; tRNA, transfer RNA. 
A
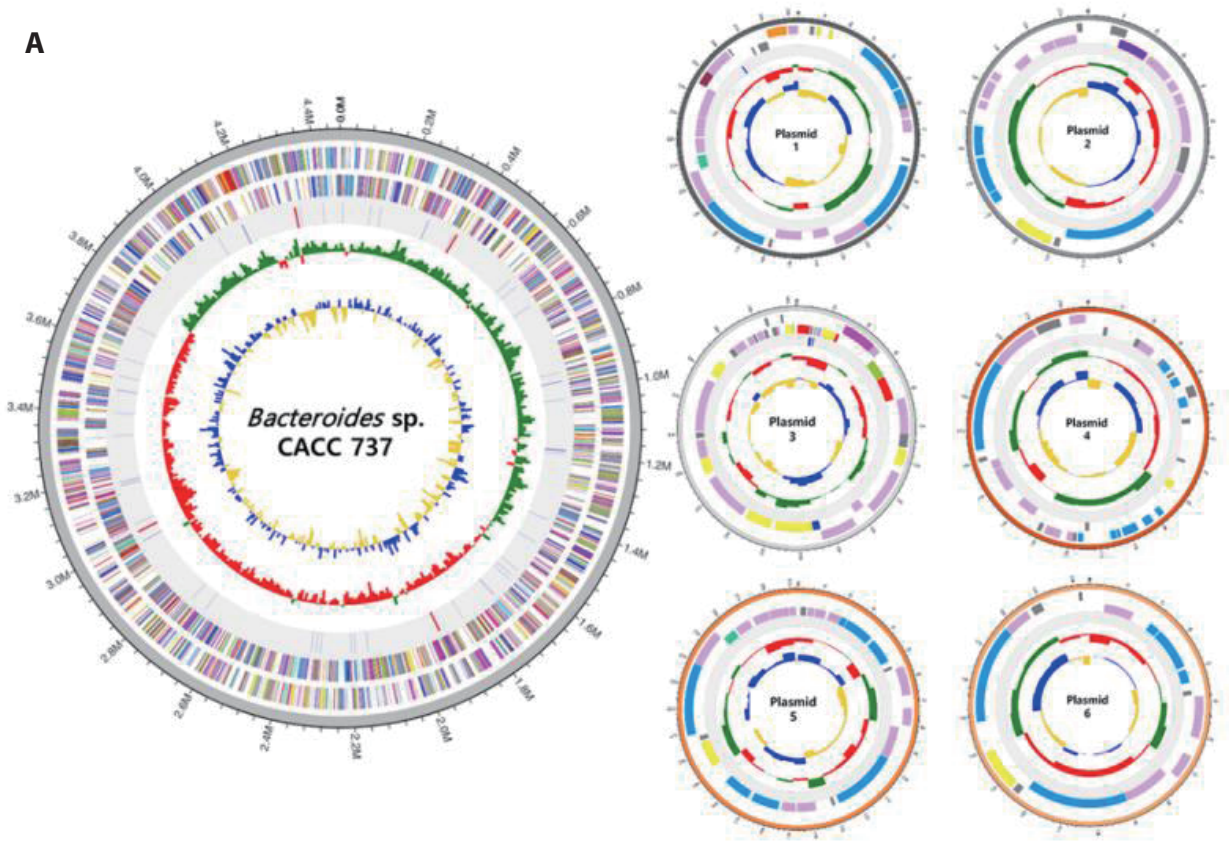

B
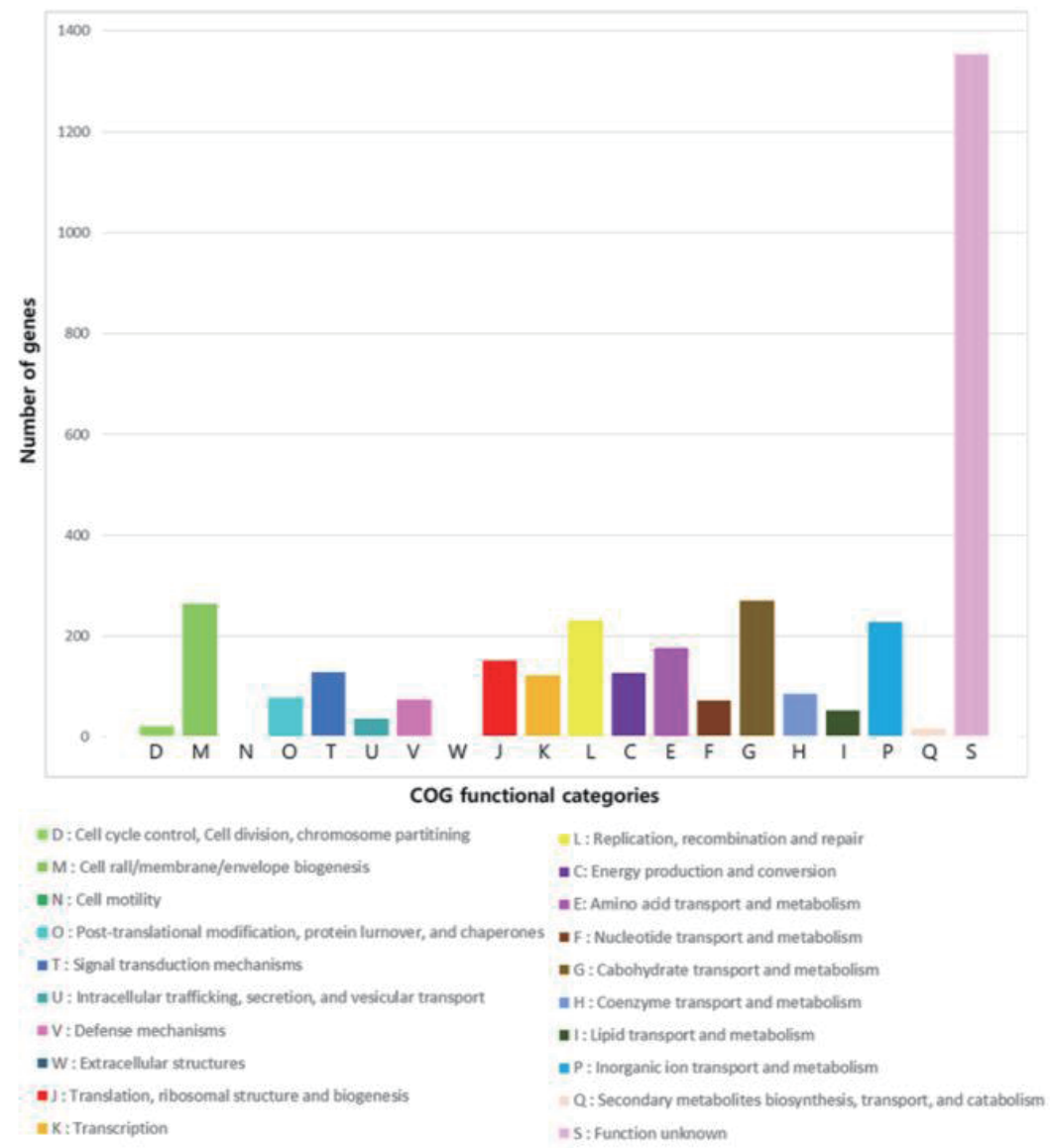

Fig. 1. Genome features of Bacteroides sp. CACC 737. (A) Circular genome maps of Bacteroides sp. CACC 737 chromosome and plasmids. Circles from the outside to the center denote rRNA and tRNA gene, reverse strand CDS, forward strand CDS, GC skew, and GC content. (B) Genome number of COG functional categories; rRNA, ribosomal RNA; tRNA, transfer RNA; COG, clusters of orthologous group; CDS, coding sequence; $\mathrm{GC}$, guanine-cytosine. 
Immunol. 2019;10:666. https://doi.org/10.3389/fimmu.2019.00666

4. Marsilio S, Pilla R, Sarawichitr B, Chow B, Hill SL, Ackermann MR, et al. Characterization of the fecal microbiome in cats with inflammatory bowel disease or alimentary small cell lymphoma. Sci Rep. 2019;9:19208. https://doi.org/10.1038/s41598-019-55691-w

5. Kim M, Oh HS, Park SC, Chun J. Towards a taxonomic coherence between average nucleotide identity and 16S rRNA gene sequence similarity for species demarcation of prokaryotes. Int J Syst Evol Microbiol. 2014;64:346-51. https://doi.org/10.1099/ijs.0.059774-0

6. Aziz RK, Bartels D, Best AA, DeJongh M, Disz T, Edwards RA, et al. The RAST server: rapid annotations using subsystems technology. BMC Genomics. 2008;9:75. https://doi. org/10.1186/1471-2164-9-75

7. Grissa I, Vergnaud G, Pourcel C. CRISPRFinder: a web tool to identify clustered regularly interspaced short palindromic repeats. Nucleic Acids Res. 2007;35 Suppl 2:W52-7. https://doi. org/10.1093/nar/gkm360

8. Arndt D, Grant JR, Marcu A, Sajed T, Pon A, Liang Y, et al. PHASTER: a better, faster version of the PHAST phage search tool. Nucleic Acids Res. 2016;44:W16-21. https://doi. org/10.1093/nar/gkw387

9. Nguyen M, Vedantam G. Mobile genetic elements in the genus Bacteroides, and their mechanism(s) of dissemination. Mobile Genet Elem. 2011;1:187-96. https://doi.org/10.4161/ mge.1.3.18448

10. Hidalgo-Cantabrana C, Crawley AB, Sanchez B, Barrangou R. Characterization and exploitation of CRISPR loci in Bifidobacterium longum. Front Microbiol. 2017;8:1851. https://doi. org/10.3389/fmicb.2017.01851 